Educación Física y Ciencia, vol. 21, nº 1, e069, enero-marzo 2019. ISSN 2314-2561

Universidad Nacional de La Plata.

Facultad de Humanidades y Ciencias de la Educación.

Departamento de Educación Física

\title{
Hacia una comprensión de la evaluación conceptual en educación física
}

\author{
Towards an understanding of the conceptual evaluation in physical education
}

\author{
Sebastián Peña Troncoso \\ Universidad de Austral de Chile y Universidad Sek, Chile \\ sebastian.pena@uach.cl
}

Sergio Toro Arévalo

Universidad de Santiago de Chile, Chile

seatoro@gmail.com

Juan Carlos Beltrán Veliz

Universidad Católica de Temuco, Chile

beltranovic@hotmail.com

\author{
Braulio Navarro Aburto \\ Universidad Autónoma de Chile, Chile \\ 002ademir@gmail.com
}

\section{RESUMEN:}

El conocimiento, en su dimensión conceptual es el tipo de "saber" que habitualmente se ha vinculado desde las diferentes asignaturas que conforman el currículo escolar, excepto en educación física. Desde esta mirada, el objetivo del presente trabajo es, comprender el sentido que le otorgan los profesores de educación física a la evaluación conceptual. Se llevó a cabo una investigación con un enfoque cualitativo y como técnica de recolección de datos la entrevista semi-estructurada; los relatos fueron analizados y categorizados bajo la técnica de análisis de contenido. Participaron doce profesores de las diferentes dependencias administrativas de la Provincia de Llanquihue, Región de los Lagos. Los resultados más relevantes evidencian, por un lado, confusión conceptual en la disciplina, enmarcada en el carácter funcional de la evaluación, medición y la calificación y, por otro, los profesores de educación física reconocen importantes beneficios de la evaluación conceptual en la disciplina, no obstante, la mayoría de los docentes entrevistados continúan realizando evaluaciones orientadas a la dimensión procedimental (saber hacer). En tal sentido es posible concluir, la existencia de un encasillamiento de la evaluación con fines orientadores al control de objetivos y aprendizajes. Palabras ClaVe: Evaluación, Educación Física, Profesores.

\section{Abstract:}

Knowledge, in its conceptual dimension, is the type of "knowledge" that has usually been linked from the different subjects that make up the school curriculum, except in Physical Education. From this perspective, the objective of this paper is to understand the meaning that Physical Education teachers give to conceptual evaluation. An investigation was carried out with a qualitative approach and as a data collection technique, the semi-structured interview was performed. The stories were analyzed and categorized under the content analysis technique. Twelve teachers from different administrative units of Llanquihue's Province, Region of Los Lagos, took part. The most relevant results show, on the one hand, conceptual confusion in the discipline, framed in the functional character of the evaluation, measurement and qualification and, on the other, Physical Education teachers recognize important benefits of the conceptual evaluation in the discipline. Nevertheless, most of the teachers interviewed continue to carry out evaluations oriented towards the procedural dimension (know-how). In this sense, it is possible to conclude the existence of a classification of the evaluation with aims pointed at controlling objectives and learning.

KEYWORDS: Evaluation, Physical education, Teachers.

\section{INTRODUCCIÓN}

El estudio forma parte importante en el desarrollo de la tesis doctoral del autor principal, el cual pone de manifiesto que, la evaluación en Educación Física (EF), debe considerar los diferentes elementos del currículo; conceptos, actitudes y procedimientos, de tal forma, que asegure que se valora tanto el grado 
de adquisición de las capacidades básicas, como el grado de consecución de los objetivos. Uno de estos elementos son los contenidos, los cuales para asegurar su tratamiento de forma integral, deben de atender a contenidos relacionados con el conocimiento teórico y práctico (Arnold, 1991) denominados como contenidos conceptuales; contenidos basados en procesos y en la propia ejecución, denominados como contenidos procedimentales o de saber práctico (Navarro y Jiménez, 2009); y contenidos que representen una adhesión a unos valores y normas determinadas, denominados como contenidos actitudinales (Blázquez, 2010).

Cuando revisamos las diferentes evaluaciones que existen en $\mathrm{EF}$, podemos visualizar que existe una tendencia hacia la evaluación procedimental (saber hacer) a través de baterías motrices y test físicos, dejando en un segundo plano la dimensión del conocimiento conceptual(Peña-Troncoso, Toro, Osses, Pachón, \& Hernández-Mosqueira, 2019).Para Anderson (1987) dicho conocimiento lo define como el conjunto de atributos y características que decimos de un objeto, suceso o idea. Se identifica con el "saber", siendo la información que podemos recordar exactamente como fue memorizada (McPherson, 1994; Thomas y Thomas, 1994). La característica principal de este tipo de conocimiento es que el alumno lo puede verbalizar y declarar (Ruiz, Sánchez, Durán, y Jiménez, 2006) o que se conforma por medio del lenguaje (Díaz-Barriga y Hernández, 2002).

Diferentes estudios (Carmona, 2007; Domínguez, Arroyo, Gallego, González, y del Villar, 2006; Hernández, Velázquez, y Martínez, 2007); Ibañez, 2005; Peña y Hernández, 2014; Ponce, 2005; Otero, González, Calvo, y Molina, 2012; Velázquez, Hernández, Martínez, y Martínez de Haro, 2011) señalan que la evaluación en educación física se ha centrado tradicionalmente en los contenidos procedimentales, dicho de otra manera preocupados del "saber hacer", aun cuando el conocimiento conceptual es crucial para el perfeccionamiento del proceso pedagógico en el ámbito del entrenamiento de niños y jóvenes (Aleixo y Mesquita, 2016).

\subsection{La evaluación en educación}

La evaluación en educación es quizás, uno de los temas más relevantes y trascendentes dentro del desarrollo curricular de cualquier sistema escolar. Constantemente los docentes están evaluando a sus alumnos, cada profesor evalúa su asignatura, los alumnos son evaluados, los docentes son evaluados, los alumnos evalúan a sus compañeros, evalúan a sus profesores, se autoevalúan y en algunos casos se evalúan los procesos. Sin duda, todos los actores que componen el sistema educativo están inmersos en constantes procesos de evaluación. En un sentido amplio, la evaluación en educación consiste "en un proceso de delinear, obtener, procesar y proveer información válida, confiable y oportuna sobre el mérito del aprendizaje de un estudiante con el fin de emitir un juicio de valor que permita tomar diversos tipo de decisiones" (Ahumada, 2003). Sin embargo, también es muy importante considerar en la evaluación en educación, el proceso de enseñanza y aprendizaje y el propio proceso del profesor.

Es importante hacer una distinción hacia la propia evaluación educativa, ya que esta, alude al tipo de evaluación que se realiza dentro de los procesos de enseñanza y aprendizaje en el aula, en las situaciones educativas formales y pertenecientes al mundo escolar, no estamos hablando de la evaluación entendida como "evaluación de programas" (aunque sean educativos), o como investigación evaluativa” (López, 2006, p. 21). Con esto apunta a la idea de evitar ciertas confusiones que se producen al hablar de evaluación educativa, porque puede estar dirigida con fines distintos según la aproximación que se haga de ella. Si bien existen muchos autores que hacen referencia a la evaluación, y generan una amplia mirada en torno a ésta, hay aspectos coincidentes.

Para Castejón (2007), la evaluación puede entenderse como la valoración que se realiza de un hecho observado de acuerdo a unos datos obtenidos por algún medio definido. Es importante que se atienda al término "valoración", ya que la evaluación siempre debe dar una opinión y esta será más o menos objetiva 
dependiendo de la medición que se haya realizado. La evaluación en educación es difícil de asumir como objetiva y es más apropiado decir que la evaluación es una valoración subjetiva. En esta línea, Santos Guerra (1993), conceptualiza a la evaluación como un proceso de indagación sobre el valor educativo, en donde existe un proceso de dialogo, comprensión y mejora permanente. Pero siempre considerando las tres dimensiones del conocimiento (conceptual, actitudinal y procedimental).

\subsection{La evaluación en educación física}

En los últimos años la evaluación en $\mathrm{EF}$ ha sido tema de conversación en los diferentes marcos curriculares, principalmente, porque se busca reemplazar la visión reduccionista de la evaluación del rendimiento, por una evaluación que abarque el conjunto de aspectos, procesos y variables involucradas en las actividades escolares, en definitiva, se intenta llegar a una mirada integral de la evaluación en EF.

En Chile, por ejemplo, el Sistema de Medición de la Calidad de la Educación (SIMCE) en Educación Física, ha sido estos últimos años la herramienta de evaluación nacional en la asignatura de EF. La prueba está basada en la conocida batería de test Eurofit y es aplicada a los estudiantes de octavo año básico (13-14 años), en un intento por conocer la condición física de los estudiantes chilenos (Agencia de la Calidad de la Educación, 2013). Este tipo de medición ha llevado a la disciplina a un parcelamiento del conocimiento, orientando todo a través del saber hacer. Asimismo, esta situación hallevado a una conceptualización errónea que tiene la población frente a la educación física (Moreno, Campos, y Almonacid, 2012). Y lo que es peor aún, los directivos de los establecimientos educacionales se están interesando por la EF, al extremo de solicitar a los docentes que incrementen los resultados de las pruebas físicas en los menores para que logren figurar en el ranking nacional con altos resultados en el SIMCE en EF, continuando de esta manera, con la mirada de una asignatura mecanicista y tecnocrática orientada a evaluar la condición física y algunas variables antropométricas (Moreno y Medina, 2012), que por lo demás, tienen un bajo impacto para los estudiantes en sus procesos de enseñanza y aprendizaje (Peña, Osses, Navarro y Beltrán, 2017).Aun así a los colegios les interesa poder obtener buenos resultados en la medición (Rodríguez, Coz, Durán, Guajardo, Alvarado, y Doña, 2015). Autores como Rodríguez-Rodríguez, Curilem, Escobar, y Valenzuela (2016) señalan que la disciplina necesita una evaluación integral y esta debe abarcar instrumentos relacionadas con la condición física, agilidad y coordinación, habilidades motoras abiertas y expresión motriz. Sin embargo, este tipo de evaluación, continúa fragmentando el conocimiento, al no considerar los conocimientos y las actitudes.

Existen varios factores que pueden explicar, en buena parte, el hecho de que la EF continúe ignorando los contenidos de carácter conceptual y, por tanto, la exigencia de los correspondientes aprendizajes. Uno de ellos lo constituye la escasa asignación horaria semanal que han tenido los docentes en su práctica laboral. El otro, posiblemente de mayor peso, radica en que la concepción hegemónica de esta disciplina que le atribuye al desarrollo de la competencia motriz del alumnado su razón de ser y su objeto principal, lo que supone tratar de obtener el mayor tiempo posible de compromiso motor durante el desarrollo de las clases (Hernández, Velásquez, y Martínez, 2007). En efecto, la acción conjunta de ambos factores ha determinado que, a lo largo de la historia de la EF, la preocupación de los profesores e investigadores haya estado centrada siempre en la práctica motriz, orientados al "rendimiento deportivo" (González, García, Pastor y Contreras, 2011; Méndez, 2005) y más concretamente, en la variable "tiempo de aprendizaje" (ALT-PE: Academic Learning Time in Physical Education) (Gusthart, Kelly \& Rink, 1997; Metzler, 1989; Silverman, Devillier \& Ramírez, 1991).

Si bien, en educación física se asume rara vez una evaluación integral desde las tres dimensiones que construyen el conocimiento (conceptual, procedimental y actitudinal), estos aún siguen estando explícito en todos los programas de estudio de EF,e incluso en los estándares orientadores para la calidad de la Educación Física y Salud de la Formación Inicial Docente (FID) de Chile (Mineduc, 2013). 
Zabala, Viciana y Lozano (2002) señalan que, los profesores priorizan el aprendizaje, otorgando una clara importancia a los procedimientos sobre los conceptos y actitudes. En esta línea, López y Moreno (2002) en un análisis de la enseñanza de estos contenidos en EF señalan que uno de los problemas de los docentes del área, es el desconocimiento de cómo llevar los conocimientos teóricos a las clases de EF. Señalan una importante deficiencia en la "formación inicial" como causa, y definieron tres perfiles docentes en relación con este aspecto. En primer lugar, aquellos que afrontan su incapacidad con un inmovilismo basado en continuar con la metodología tradicional. Otra corriente basa el aprendizaje de hechos y conceptos en las clases teóricas y evaluaciones escritas. La tercera tendencia fundamenta el proceso de enseñanza y aprendizaje conceptual en los libros de texto.

Finalmente, la mejora de la calidad de las clases es importante y no puede perderse el objetivo de la disciplina, orientándola sólo al rendimiento deportivo (Flintoff, Foster, \& Wystawnoha, 2011), sino que, además, debe existir un impacto desde lo cualitativo en la formación de los estudiantes y profesores.

\subsection{La evaluación conceptual en educación física}

Los contenidos conceptuales continúan constituyendo el tipo de saber que habitualmente se ha transmitido desde las diferentes disciplinas escolares (Pozo, 1999). A pesar de lo expresado por este autor, este no es el caso de la EF. Esta disciplina escolar se ha centrado tradicionalmente en la mejora de la habilidad motriz y la condición física, es decir, se ha caracterizado por un predominio del "saber hacer" y por una pobreza manifiesta en cuanto a la presencia y transmisión de conocimiento conceptual (Velázquez, et al., 2011).

Desde una perspectiva más general, Hernández \& López (2007) señalan que los conocimientos conceptuales han sido, y aún continúan siendo, el tipo de contenidos que ha merecido mayor atención en la transmisión del "saber" desde las diferentes áreas curriculares. Es decir, en el sentido de aquel "saber" que permite que los estudiantes sean capaces de comprender, fundamentar y explicar sus propias acciones. Desde esta mirada, Velázquez, et al., (2011) señalan que es aconsejable la inclusión de contenidos conceptuales en los currículos de EF por los potenciales efectos que tendría su aprendizaje con respecto a la adopción de unos u otros estilos de vida. No obstante, nos surge una interrogante ¿La Educación Física podrá alcanzar estos aprendizajes en sus estudiantes, sin promover el conocimiento conceptual? difícilmente los alumnos podrán ser capaces de comprender, dar significado y de prever las consecuencias de las actividades que se realizan, formarse opiniones propias basadas en el conocimiento y en la razón, o planificar de forma autónoma planes personales de actividad física- deportiva coherente y saludable (Hernández \& López 2007). Desde una concepción interpretativa y crítica de los contenidos conceptuales, la formación de estos permitirá que las jóvenes generaciones construyan significados vinculados al cuerpo y a la actividad física. Pérez (2000) señala que, "existen múltiples factores que desencadenan los procesos de construcción de significados" y, cabe afirmar que, uno de esos factores lo constituye el conocimiento y la comprensión de conceptos básicos capaces de contribuir a que el sujeto interprete y participe de su propia realidad social. La capacidad de tomar decisiones, de emitir juicios de valor autónomos, críticos y razonados, de elaborar planes de acción relacionados con las necesidades de actividad física y con su empleo en el tiempo libre, el cual requiere disponer de los conocimientos (hechos, principios, conceptos) que permiten fundamentar con un mínimo de coherencia y rigor las decisiones y acciones personales. De igual modo, la adquisición de tal tipo de conocimientos es básica para la formación del estudiante como un espectador responsable y capaz de comprender y valorar de manera ponderada ese bien cultural que, en la actualidad, suponen los eventos deportivos en nuestra sociedad ( Hernández, Velásquez, y Martínez, 2007).

Desde la EF, se consideran importantes los procedimientos o habilidades en las clases, pero, la crítica apunta a la necesidad de valorar el conocimiento disciplinar y actitudinal de la disciplina, porque la experiencia del aprendizaje en EF debe nacer desde la incorporación de conocimiento hacia la ejecución, y a partir de una actitud, determinada por las situaciones o problemas que se enfrentan (Zabala, 2000). De 
esta manera, el instante más relevante del desarrollo intelectual se produce cuando lo teórico (contenidos conceptuales) converge con lo práctico y la experiencia (Castañer \& Trigo, 1995; Toro, Pazos, Vargas, \& Vega, 2016), sin invisibilizar, los valores y las actitudes en cada acción desde el mismo proceso de aprendizaje. En razón de los antecedentes y de la problemática expuesta, se plantea como objetivo, comprender el sentido que le otorgan los profesores de educación física a la evaluación conceptual.

\section{Metodología}

La metodología utilizada en la presente investigación es de carácter cualitativo descriptivo y con un enfoque hermenéutico, puesto que tiene relevancia específica para el estudio de las relaciones sociales, debido al hecho de la pluralización de los mundos vitales (Flick, 2004). Para la recolección de los datos se utilizó la entrevista semi-estructurada, elaborada a partir de un guión que determino cumplir con nuestro objeto de estudio. "Es una modalidad que permite ir entrelazando temas e ir construyendo un conocimiento holístico y comprensivo de la realidad" (Bisquerra et al., 2012, p.337).En este sentido, el guión de preguntas de la entrevista semi-estructurada son flexibles, es decir, el investigador va adecuando las preguntas de acuerdo a lo que se quiere indagar. Esta técnica permitió comprender el sentido que le otorgan los profesores de educación física a la evaluación conceptual de la disciplina.

La aplicación de las entrevistas, fueron realizadas por los propios investigadores, considerando, primero, entrar en un clima de confianza con los profesores, dándole todas las facilidades y comodidades posibles para la recogida de información y, segundo, poder apreciar en aquellas respuestas las posibilidades de explorar más profundamente la importancia que le otorgan a los contenidos conceptuales en EF.

Es importante señalar que para cumplir con los criterios éticos propuestos, hemos de resguardar el anonimato de todos los participantes, utilizando nombres alternativos. Los profesores, también debieron firmar un consentimiento informado antes de la entrevista. Al respecto, Taylor y Bogdan (1987) señalan que, el ingreso en un escenario generalmente implica una especie de pacto: la seguridad implícita o explícita de que no se desea violar la privacidad o confidencialidad de los informantes, ni exponerlos a perjuicios, ni interferir en sus actividades. Con ello, se respetaron los criterios éticos, de acuerdo a la declaración de Helsinki para el estudio con seres humanos (Declaración de Helsinki de la Asociación Médica Mundial, 2008). En este contexto, el estudio fue aprobado por el Comité Ético Científico (CEC) de la Universidad de la Frontera, Chile.

\subsection{Participantes}

El estudio se realizó con profesores de EF de la Provincia de Llanquihue, Región de los Lagos, de las tres dependencias educativas (municipales, subvencionadas y particulares). Participaron doce docentes, los cuales fueron seleccionados intencionalmente como parte de un estudio doctoral del autor. Al respecto, Gurdián (2010, p. 247) señala que, la selección apropiada de las y los sujetos actuantes, permite un mejor conocimiento del fenómeno por investigar. Esto garantiza una saturación efectiva y eficiente de las categorías con información de óptima calidad. En este contexto, se presenta a continuación los criterios de inclusión en la tabla 1. 
TABLA 1.

Criterios de inclusión de los participantes

\begin{tabular}{|l|l|}
\hline Criterios & Descripción \\
\hline Género & Femenino y masculino \\
\hline Rol o profesión que desempeña & Profesor/a de educación secundaria \\
\hline Ciclo & Tercer ciclo: séptimo a cuarto de secundaria \\
\hline Ex periencia profesional & Minimo 3 años en aula \\
\hline Contexto & Particular, subvencionado y municipal \\
\hline
\end{tabular}

Fuente: Elaboración propia (2018).

\subsection{Análisis de los datos}

Los datos se analizaron bajo la técnica de análisis de contenido, a través de un análisis secuencial de los discursos (Potter y Wetherell, 1998). Con el propósito de resumir los relatos de carácter particular de las entrevistas, se buscaron los conceptos descriptivos de un nivel superior y se optó por las categorías y sub-categorías de análisis (Pochet, 2000; Valles, 1999). Para ello, se utilizó el software Atlas Ti. versión 7.5. Este software agiliza de forma considerable las actividades implicadas en el proceso de análisis de las entrevistas, como la segmentación del texto en citas, el proceso mismo de codificación, la escritura de memos y anotaciones, y la realización de vínculos entre citas, memos y códigos (Flores, 2009).En tal sentido, se levantaron categorías y subcategorías, para luego identificar las relaciones entre estas y desarrollar una red conceptual. Posteriormente, se realiza la discusión de resultados por categoría y sub-categoría.

\section{Discusión de RESUltados}

Desde el análisis de contenido emergieron dos categorías principales: 1)Evaluación en Educación Física, de la cual se desplegaron cuatro sub-categorías: a) Medición, b) Instrumento de control, c) Proceso continuo y, d) Proceso Formativo. La segunda: 2) Evaluación conceptual, de la cual forman parte tres sub-categorías: a) Cultura, b) Desarrollo Integral y, c) Transversalidad.

FIGURA 1.

Sub-categorías que forman parte de la categoría evaluación en educación física

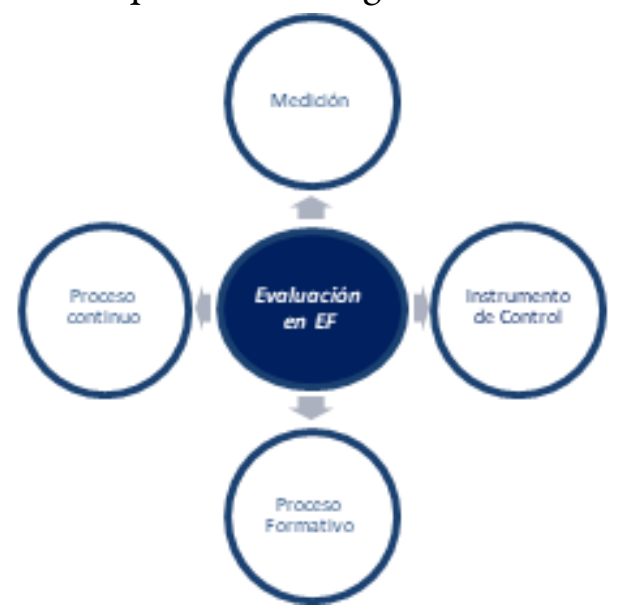

Fuente: Elaboración propia(2018) 


\section{Categoría 1.Evaluación en educación física}

En esta categoría se devela que no existe igualdad de opinión por parte de los profesores de educación física en torno al significado de la evaluación en el contexto educativo. De esta manera se visualizan diferentes formas de entenderla.

Esto se ve reflejado en discurso:"La evaluación es un proceso, en realidad es un momento de un proceso de aprendizaje, en esta evaluación puede estar incluida en cualquier momento del proceso, tanto en el principio durante o posterior, es una evaluación participante, es decir que los alumnos y el evaluador son activos y conscientes en los resultados, y la evaluación busca de igual manera como dar respuesta a todas las áreas que el profesor planteó en un principio en la unidad de aprendizaje" (E: 1).

Al respecto Hernández y Velázquez (2004) declaran que evaluar, en un sentido amplio, supone la realización de un conjunto de acciones encaminadas a la obtención de información significativa con el fin de, una vez analizada e interpretada en un marco de referencia, hacer posible la elaboración de un juicio de valor y, si procede, una toma de decisiones sobre los diferentes elementos y factores que configuran e interactúan en el sistema educativo. En este sentido, López (2006) comenta que, entendiendo de esta forma la evaluación, se puede evidenciar claramente que la mayoría de las actividades denominadas de evaluación son simples procesos de calificación. Es decir, la evaluación está enfocada en un momento, específicamente en los resultados.

\section{Sub-categoría a). Instrumento de control}

En la mayoría de los discursos de los profesores de EF, se visualiza una orientación de la evaluación hacia un producto o un objetivo. Así lo reflejan los siguientes fragmentos: "La evaluación para mí en el fondo es como un instrumento control porque me ayuda a saber que están aprendiendo los chicos de lo que yo le entrego y que tan efectivo está siendo entregado los conocimientos hacia ellos"(E: 6). En continuidad: "A mí me sirve la evaluación como instrumento de control, independiente del resultado que obtengan los estudiantes en cada una de ellas" (E: 3).

Desde esta mirada, podemos visualizar que los profesores de EF aún mantienen una mirada de la evaluación asociada a la racionalidad técnica basada en el control. Esta visión responde a una mirada sesgada antes del $\mathrm{XX}$, donde la única finalidad de la evaluación era medir los aprendizajes de los alumnos a través de la conducta, es decir, como pedagogía o programación por objetivos (López, 2009; Díaz, 2005).

\section{Sub-categoría b). Medición}

En educación física, la medición es quizás uno de los términos con el cual más se confunde la evaluación, condicionado muchas veces por la cantidad de test físicos que se aplican en la disciplina.

Es así, como se evidencia en las siguientes narraciones: "La evaluación para mi es lograr medir el rendimiento físico de los alumnos, para saber cómo se encuentran” (E: 7). En correspondencia, otro entrevistado declara: "Para mí, la evaluación es como un instrumento de medición de los aprendizajes de los estudiantes, independientemente del resultado que tenga la evaluación” (E: 9).

Según Blázquez (2010) la medición es la acción orientada a la obtención y registro de información cuantitativa (expresa en número su cantidad o grado) sobre cualquier hecho o comportamiento. Desde esta mirada, si analizamos las impresiones de los profesores, se aprecia la gran influencia hacia una evaluación 
tradicional, donde los alumnos no son capaces de involucrarse de forma autónoma en sus procesos de aprendizaje y solo son objetos de medición.

\section{Sub-categoria c). Proceso continúo}

La evaluación como proceso continuo, es otro de los términos recurrentes que los profesores de educación física no logran clarificar. Así lo reflejan las siguientes narraciones: "Para mí la evaluación es un proceso continuo, ya que definitivamente lo que menos me interesa es el resultado final" (E:7). Lo anterior, se complementa con en el siguiente relato: "Para mí la evaluación es un proceso continuo, en donde los niños van construyendo a la par los conocimientos que van aprendiendo y los conocimientos que los van formando para la vida" (E:4).

$\mathrm{Al}$ respecto, Castejón (2007) declara que la evaluación continua, por su carácter formativo, supone una constatación de que el estudiante desarrolla y aplica elementos de los diferentes ámbitos cognitivo, afectivo-social y motriz. En síntesis, la evaluación continua, está orientada al momento en el cual se realiza la evaluación, ya que esta puede ser realizada en diferentes momentos del proceso de evaluación. En complementación, Ahumada (2005) señala que la evaluación debe estar centrada en la continuidad y permanencia, más que en un momento, y que debe tener un carácter retroalimentador.

\section{Sub-categoria d). Proceso Formativo}

La evaluación formativa corresponde a una visión de la enseñanza en la que se considera que aprender es un proceso del cual los estudiantes son parte de su propio proceso de enseñanza y aprendizaje. Así, lo develan los discursos de algunos docentes entrevistados:

"La perspectiva que hoy tengo, es más llevado a un proceso formativo, en donde más que mediciones, el sujeto pueda conocer y superar sus dificultades a partir de él mismo y no de los otros o el mejor desempeño de los otros” (E:10).

Este modelo de evaluación ha sido motivo de reflexión en los últimos años en el contexto educativo. Autores como (Díaz, 2005; López, 2009; Velázquez \& Hernández, 2004) señalan que la evaluación adquiere un carácter formativo cuando se utiliza para intentar mejorar el proceso de enseñanza y aprendizaje a partir del conocimiento que genera de dicho proceso. En síntesis, este tipo de evaluación nos permite regular de mejor forma los procesos de enseñanza y aprendizaje de las diferentes unidades didácticas, logrando asegurarnos, por un lado, que las características de los alumnos respondan a las exigencias del sistema y, por otra, garantizar que los medios de formación se correspondan con las características de los alumnos. Sin duda, no sólo nos permitirá realizar una evaluación mucho más objetiva, sino que además los alumnos pueden ser parte de su propio proceso de enseñanza y aprendizaje. 
FIGURA 2.

Sub-categorías que forman parte de la categoría evaluación conceptual

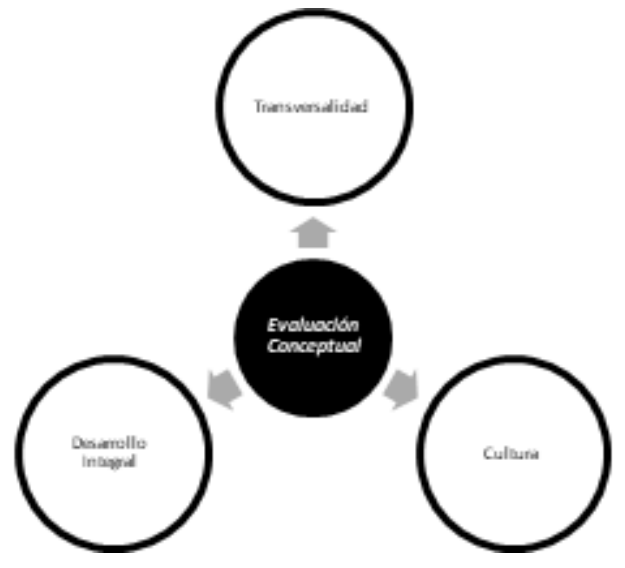

Fuente: Elaboración propia (2018)

\section{Categoría 2. Evaluación conceptual}

La evaluación conceptual, quizás ha sido la dimensión del conocimiento que merecido mayor atención desde la educación física, principalmente, porque se considera en escasa ocasiones en la evaluación integral. Los relatos de los diferentes profesores entrevistados develan por ejemplo: "Yo le doy bastante importancia a los contenidos conceptuales, porque me permite preparar de mejor manera a los estudiantes y, así, puedan tener conocimientos básicos, por ejemplo; anatomía, temas de fisiología, temas propios del entrenamiento...”(E:10). No obstante, otras narraciones hacen alusión a otra mirada de la evaluación conceptual: "Creo que son importantes de todas maneras, pero hasta el momento nosotros no evaluamos esa parte, es decir, lo podemos comentar, podemos tener conversaciones a medida que vamos haciendo las cosas, le vamos dando contenidos, pero no lo evaluamos, no tenemos evaluación de ese tipo" (E:8).

Sin duda, existe una dualidad de opinión cuando se trata de hablar de los contenidos conceptuales, aun estando explícitos en los programas de estudio del sector de educación física. López \& Moreno (2002), en un análisis de la enseñanza de estos contenidos en educación física señalan que uno de los problemas de los docentes del área es el desconocimiento de cómo llevar los conocimientos conceptuales a las clases de EF. A modo de síntesis, es innegable reconocer que la predominancia sigue estando constituida por los aprendizajes procedimentales "saber hacer".

\section{Sub-categoria a). Transversalidad}

La transversalidad busca mirar toda la experiencia escolar como una oportunidad para que los aprendizajes integren sus dimensiones cognitivas y formativas, y así, lograr impactar en el currículum, en la cultura escolar y en todos los actores que forman parte de ella. Los discursos de los profesores en torno a la importancia de la evaluación conceptual señalan: "La importancia siempre estará en los contenidos procedimentales, pero, los contenidos conceptuales nos permiten transversalidad, con varias asignaturas..." (E:1). Esta conexión, sin duda, enriquece los procesos de enseñanza y aprendizaje de los estudiantes.

Para Reyzábal \& Sanz (1995), la transversalidad educativa enriquece la labor formativa de manera tal que conecta y articula los saberes de los distintos sectores de aprendizaje y dota de sentido a los aprendizajes disciplinares, estableciéndose conexiones entre lo instructivo y lo formativo. 
A modo de reflexión, el término de transversalidad es una gran oportunidad de seguir creciendo en el contexto educativo, para lograr contribuir a los aprendizajes significativos de los estudiantes desde la conexión de los conocimientos disciplinares con los temas y contextos sociales, culturales y éticos presentes en su entorno.

\section{Sub-categoria b). Cultura}

Varios de los discursos de los profesores de educación física entrevistados, declaran la importancia de la evaluación conceptual como cultura esencial en sus procesos de enseanza y aprendizaje. Algunos de los relatos sealan: "Creo que son importantes los contenidos conceptuales como cultura general, dado que los estudiantes deben conocer cómo funciona su cuerpo en relación a la actividad física" (E: 9). Lo anterior, se complementa con en el siguiente relato: "Para nosotros como profesores, la evaluación conceptual es esencial, porque permite conocer realmente si las actividades que realizamos a diario con los estudiantes, se logran comprender”... (E: 4).

En este contexto, la cultura según (Tulviste, 1992; Wertsch, 2000) es entendida como un conjunto de escenarios de actividad definidos socioculturalmente, en los que tienen lugar el funcionamiento psicológico humano y en los que la participación en los mismos guía el desarrollo individual; es decir, se constituye en el principio explicativo de la cognición humana. Es ésta una noción de cultura que va más allá de los elementos físicos del medio, ya que todo escenario de actividad está basado en una serie de suposiciones implícitas sobre los aspectos necesarios para que dicho contexto exista y sea reconocido por sus integrantes. En síntesis, la educación física es reconocida como una disciplina vivencial, sin embargo, no debemos olvidar que el cuerpo y el movimiento son los ejes básicos en los que se centra la acción educativa. Se trata, por un lado, de mejorar el comportamiento motor de los estudiantes y, por otro, la educación a través del cuerpo y el movimiento para adquirir competencias de carácter cognitivos, afectivo y de relación, necesarias para la vida en sociedad.

\section{Sub-categoria c). Desarrollo Integral}

El sector educación física contribuye a la formación integral de la persona, razón por la cual no sólo focaliza sus esfuerzos en el desarrollo del potencial motriz de los alumnos, sino también se propone su desarrollo afectivo, social, cognitivo, moral y espiritual. Es en esta línea, varios de los docentes señalan: "Los contenidos conceptuales permiten en los estudiantes un desarrollo integral en su formación, pues está muy llevado hacia la concepción del ser, el hacer y el saber hacer..." (E: 10). Lo anterior, se vincula con el siguiente discurso: "La evaluación conceptual permite un desarrollo integral de los alumnos, ya que permite que los estudiantes logren hacer, pero también saber...” (E: 9).

Respecto a lo anterior, la evaluación conceptual busca estimular en los alumnos el desarrollo de las capacidades reflexivas y críticas que les habiliten para analizar y discutir la finalidad, consecuencias biológicas, sociales y psicológicas de cada una de las prácticas educativas, físicas, deportivas y recreativas en las que ellos se verán implicados (Mineduc, 2009). Es por ello, la importancia de integrar los contenidos conceptuales para mejorar el desarrollo integral de los estudiantes. Principalmente porque toda competencia motriz debe de manera paralela desarrollar una competencia "intelectual" que dote a los estudiantes de la capacidad de conducirse con autonomía, responsabilidad y sentido crítico en el campo de la actividad físico-deportiva.

\section{ConClusión}

Desde la discusión de los resultados respecto a la importancia de la evaluación conceptual en educación física, se concluye, por un lado, que no existe claridad en torno a la conceptualización de la evaluación en el 
contexto educativo y, por otro, se develan importantes beneficios de la evaluación conceptual en la disciplina, no obstante, la mayoría de los profesores continúan evaluando a través de metodologías tradicionales orientadas al "saber hacer", continuando de esta manera con un encasillamiento de la evaluación con fines orientadores al control de objetivos y aprendizajes.

El elemento de discusión permanente de la evaluación en educación física, se encuentra condicionada principalmente por las constantes mediciones orientadas al rendimiento deportivo de los estudiantes, es aquí, donde la medición es considerada sinónimo de evaluación y posteriormente transformada en una calificación. En tal sentido, se presenta como un proceso deshumanizador.

Estos resultados nos incitan a reflexionar sobre la necesidad de mejorar la calidad de los procesos de enseñanza y aprendizaje, con un especial enfoque en la evaluación conceptual (saber), y así, permitir que los estudiantes sean capaces de comprender, fundamentar y explicar sus propias acciones; de entender y analizar las repercusiones de dichas acciones sobre su propio cuerpo. Para ello, la evaluación debe estar centrada en un proceso continuo y permanente. Asimismo, debe contener un carácter flexible,retroalimentador, programado, dinámico, dialógico, centrado en las capacidades y estilos de aprendizajes desde una perspectiva contextualizada

\section{REFERENCIAS}

Agencia de Calidad de la Educación. (2013). Informe de Resultados SIMCE Educación Física 8o Básico para Docentes y Directivos. Santiago de Chile. Recuperado de http://www.agenciaeducacion.cl/wp-content/uploads/2013/08 /Orientaciones-B\%C3\%A1sica.pdf

Ahumada, P. (2003). La Evaluación en una concepción de Aprendizaje significativo. Ediciones Universitarias de Valparaíso. Chile (Segunda Edición). Recuperado de https://s3.amazonaws.com/academia.edu.documents/39418192/12_Ahumada_1.pdf?AWSAccessKeyId= AKIAIWOWYYGZ2Y53UL3A\&Expires=1532888415\&Signature $=$ iEtOOY7oN415wm9QBpv5JiAkKSI\% 3D\&response-content-disposition=inline\%3B\%20filename\%3D12_Ahumada_1.pdf

Ahumada, P. (2005). La evaluación auténtica: un sistema para la obtención de evidencias y vivencias de los aprendizajes. Perspectiva Educacional, Formación de Profesores, (45) 11-24.Recuperado de http://www.redalyc.org/html/333 3/333329100002/

Aleixo, I. \& Mesquita, I. (2016). Impact of implementing different teaching strategies on the development of declarative knowledge of beginner artistic gymnastics athletes. Revista Brasileira de Ciências do Esporte, 38(4), 349-357. Recuperado de http://dx.doi.org/10.1016/j.rbce.2016.01.009

Anderson, J. (1987). Skill acquisition: Compilation of weak-method problem solutions. Psychological Review, 94(2), 192-210. Recuperado de http://psycnet.apa.org/buy/1987-20973-001

Arnold, P. (1991). Educación Física, movimiento y currículum. Madrid: Morata.

Bisquerra, R., Dorio, I., Gómez, J., Latorre, A., Martínez, F., Massot, I., Mateo, J. y Vilá, R. (2012). Metodología de la investigación educativa. Madrid: La Muralla S.A

Blázquez, D. (2010). La educación fisica. Barcelona: INDE.

Carmona, R. (2007). ¿Cómo abordar los contenidos teóricos y actitudinales? Propuestas de acción en secundaria. Lecturas: Educación Física y Deportes. 104. Recuperado de http://www.efdeportes.com/efd104/contenidos-teo ricos-secundaria.htm

Castañer, M. y Trigo, E. (1995). Globalidad e interdisciplina curricular en la Enseñanza Primaria: propuestas teóricoprácticas. Barcelona: Inde.

Castejón, F. (2007). Evaluación de Programas en Ciencias de la Actividad Física. Madrid: Síntesis.

Declaración de Helsinki de la Asociación Médica Mundial (2008). Principios éticos para las investigaciones médicas en seres humano. Finlandia, Helsinki. Recuperado de https://www.wma.net/es/30publications/10policies/b3/17 c_es.pdf 
Díaz, J. (2005). La evaluación formativa como instrumento de aprendizaje en educación física. Barcelona: INDE.

Díaz-Barriga, F. \& Hernández, G. (2002). Estrategias docentes para un aprendizaje significativo. Una interpretación constructivista. México: Mc Graw Hill.

Domínguez, A., Arroyo, M., Gallego, D., González, L. \& del Villar, F. (2006). Estudio del conocimiento declarativo en función de la experiencia y de la edad en jugadores jóvenes de voleibol. Cultura, Ciencia y Deporte, 2(5), 73-80. Recuperado de http://www.redalyc.org/articulo.oa?id=163017530004

Flick, U. (2004). Introducción a la investigación cualitativa, Madrid: Morata.

Flintoff, A., Foster, R. \& Wystawnoha, S. (2011). Promoting and sustaining high quality physical education and school sport through school sport partnerships. European physical education review, 17(3), 341-351. Recuperado de ht tp://journals.sagepub.com/doi/10.1177/1356336X11416731

Flores, R. (2009). Observando Observadores: Una Introducción a las Técnicas Cualitativas de la Investigación Social. Ediciones UC.

González, S., García, L., Pastor, J., \& Contreras, O. (2011). Conocimiento táctico y la toma de decisiones en jóvenes jugadores de fútbol (10 años). Revista de Psicología del Deporte, 20(1), 79-97. Recuperado de http://www.rpdonline.com/article/view/637

Gurdián, A. (2010). El paradigma cualitativo en la investigación socio-educativa. INIE.

Gusthart, J. Kelly, I. \& Rink, J. (1997).The validity of the Qualitative Measures of Teaching Performance Scale as a Measure on Teacher Effectiveness. Journal of Teaching in Physical Education, 16(2) 196-210.Recuperado de ht tps://journals.humankinetics.com/doi/10.1123/jtpe.16.2.196

Hernández, J. \& López, A. (2007). Qué saben los escolares españoles. Conceptos claves en Educación Física y su evaluación. En J. L. Hernández y R. Velázquez (Coord.). La Educación Física, los estilos de vida y los adolescentes: cómo son, cómo se ven, qué saben y quéopinan, (pp.169-204). Barcelona: Graó.

Hernández, J. \& Velázquez, R. (coords.), (2004). La evaluación en educación física. Investigación y práctica en el ámbito escolar. Barcelona: GRAÓ.

Hernández, J. Velázquez, R. \& Martínez, M. (2007). Reformas Curriculares y Educación Física: los Conocimientos Teórico-Conceptuales de la Población Escolar Española. Archivos Analíticos de Políticas Educativas, 15. Recuperado de http://www.redalyc.org/comocitar.oa?id=275020546015

Ibañez, F. (2005). Una experiencia para el desarrollo de los contenidos conceptuales en el área de Educación Física. Lecturas: Educación Fisica y Deportes, (80), 35. Recuperado de http://www.efdeportes.com/efd80/concep.htm

López, A. \& Moreno, J. (2002). Aprendizaje de hechos y conceptos en educación física. Una propuesta metodológica. Apunts: Educación Física y Deportes, 3(69), 19-26. Recuperado de https://www.raco.cat/index.php/ApuntsEF $\mathrm{D} /$ article/view/306227

López, V. (2006). La evaluación en educación física: revisión de los modelos tradicionales y planteamiento de una alternativa: la evaluación formativa y compartida. Argentina: Miño y Dávila.

López, V. (2009). Evaluación formativa y compartida en educación superior: Propuestas, técnicas, instrumentos y experiencias. Narcea Ediciones. Madrid.

McPherson, S. (1994). The development of sport expertise: Mapping the tactical domain. Quest, 46(2), 223-240. Recuperado de https://www.tandfonline.com/doi/abs/10.1080/00336297.1994.10484123

Méndez, A. (2005). Hacia una evaluación de los aprendizajes consecuente con los modelos alternativos de iniciación deportiva. Tándem: Didáctica de la Educación Física, (17), 38-58. Recuperado de https://www.researchgate.net/profile/Antonio_Mendez-Gimenez/publication/39211980_Hacia_una_eva luacion_de_los_aprendizajes_consecuente_con_los_modelos_alternativos_de_iniciacion_deportiva/links/00 463516c57d30ef29000000/Hacia-una-evaluacion-de-los-aprendizajes-consecuente-con-los-modelos-alternati vos-de-iniciacion-deportiva.pdf

Metzler, M. (1989). A review of research on time in sport pedagogy. Journal of Teaching in Physical Education, 8(2), 87-103. Recuperado de https://journals.humankinetics.com/doi/abs/10.1123/jtpe.8.2.87 
Mineduc (2013). Estándares orientadores en educación fisica para carreras de pedagogía. Ministerio de Educación. Santiago de Chile, Chile. Recuperado de http://www.portales.mineduc.cl/usuarios/cpeip/File/nuevos\%20esta ndares/educacionfisica.pdf

Mineduc (2009). Decreto Supremo de Educación $N^{\circ}$ 220: objetivos fundamentales y contenidos minimos obligatorios enseñanza media. Santiago: Ministerio de Educación. Recuperado de http://www.agenciaeducacion.cl/wp-con tent/uploads/2013/02/Marco-Curricular-y-Actualizacion-2009-I-a-IV-Medio.pdf

Moreno, A. y Medina, J. M. (2012). Escuela educación física y transformación social. Estudios pedagógicos, 38, 7-11. Recuperado de http://mingaonline.uach.cl/scielo.php?pid=S0718-07052012000300001\&script=sci_arttext

Moreno, A., Campos, M., \&Almonacid, A. (2012). Las funciones de la educación física escolar: una mirada centrada en la justicia social y la reconstrucción del conocimiento. Estudios pedagógicos, 38, 13-26. Recuperado de https:/ /scielo.conicyt.cl/scielo.php?pid=S0718-07052012000400002\&script $=$ sci_abstract

Navarro, V., \& Jiménez, F. (2009). El conocimiento práctico de la educación física desde una visión epistemológica actual. Acción motriz. (3), 25-32. Recuperado de http://www.accionmotriz.com/documentos/revistas/articul os/3_3.pdf

Otero, F. González, J. Calvo, A. \& Molina, E. (2012). Contenidos conceptuales en Educación Física: efecto de un programa de intervención en el primer ciclo de primaria. Revista Digital de Educación Física, (16), 55-68. Recuperado de https://dialnet.unirioja.es/servlet/articulo?codigo $=3929976$

Peña, S. \& Hernández, J. (2014). Evaluación del conocimiento teórico-conceptual en Educación Física en escolares Chilenos de enseñanza media de la ciudad de Puerto Montt. Revista horizonte ciencias actividad fisica, (5), 65-82. Recuperado de http://www.revistahorizonte.ulagos.cl/index.php/horizonte/article/view/19

Peña, S., Osses, S., Navarro, B. \& Beltrán, J. (2017). Hacia la disminución de los test físicos como indicadores de salud en el contexto educativo. Salud Pública de México, 59(5), 506-507. Recuperado de https://www.scielosp.org/ar ticle/ssm/content/raw/?resource_ssm_path=/media/assets/spm/v59n5/0036-3634-spm-59-05-00506.pdf

Peña-Troncoso, S., Toro, S., Osses, S., Pachón, J. y Hernández-Mosqueira, C. (2019). La Dimensión Conceptual del Conocimiento en Educación Física: Un estudio Binacional. Retos: nuevas tendencias en educación física, deporte y recreación, (35), 170-175.

Pérez, A. (2000). La cultura escolar en la sociedad neoliberal. Madrid: Morata.

Pochet, R. (2000). La evaluación temática como una forma de análisis. Recuperado de: Discurso y análisis social: Métodos cualitativos y técnicas de análisis. (1 edición). San José, Costa Rica: Editorial de la Universidad de Costa Rica.

Ponce, F. (2005). Una experiencia para el desarrollo de los contenidos conceptuales en el área de Educación Física. Lecturas: Educación Física y Deportes, (80), 35. Recuperado de http://www.efdeportes.com/efd80/concep.htm

Potter, J. \& Wetherell, M. (1998). Social representations, discourse analysis, and racism. The psychology of the social, 138-155.Recuperado de https://books.google.cl/books? $\mathrm{hl}=\mathrm{es} \& \mathrm{lr}=\& \mathrm{id}=\mathrm{Nvz} 5 Y 8 \mathrm{e} 1 \mathrm{~N} 84 \mathrm{C} \& \mathrm{ri}=\mathrm{fnd} \& \mathrm{pg}=\mathrm{PA} 138 \& \mathrm{dq}=$ Potter, $+\mathrm{J} .,+\% 26+$ Wetherell,+M.+(1998). + Social+representations, +discourse+analysis, +and+racism. +The+psychology+of +the+social, $+138-155 . \& o t s=S w O K X 8 q 7 P n \& s i g=v x A D I 1 c S n k a z U F \_R c 8 j k I N D Y p R U \# v=o n e p a g e \& q \& f=f a l s e$

Pozo, J. (1999). Aprendizaje de contenidos y desarrollo de capacidades en la educación secundaria. En C. Coll (Coord.). Psicología de la Instrucción: la enseñanza y el aprendizaje en la Educación Secundaria. Barcelona: Horsori.

Reyzábal, M., \& Sanz, A. I. (1995). Los ejes transversales: aprendizajes para la vida. Escuela Española.

Rodríguez, F., Coz, D., Durán, T., Guajardo, A., Alvarado, C. y Doña A. (2015). Sistema de medición de la calidad de la educación física en Chile y su influencia en la realidad escolar. Movimento, 21(2): 435-448. Recuperado de h ttp://www.redalyc.org/pdf/1153/115339561011.pdf

Rodríguez-Rodríguez, F., Curilem, C., Escobar, D. \& Valenzuela, L. (2016). Propuesta de evaluación de la educación física escolar en Chile. Educación Física y Ciencia, 18(1), 00-00. Recuperado de http://www.scielo.org.ar/scielo .php?script=sci_arttext\&pid=S2314-25612016000100003

Ruiz, L., Sánchez, M., Durán, J. y Jiménez, C. (2006). Los expertos en el deporte: su estudio y análisis desde una perspectiva psicológica. Anales de psicología, 1 (22), 134- 142. Recuperado de http://www.redalyc.org/html/1 $67 / 16722117 /$ 
Santos, M. (1993). La evaluación: un proceso de dialogo, comprensión y mejora. Malaga: Aljibe.

Silverman, S., Devillier, R. \& Ramírez, T. (1991). The validity of academic learning time physical education (ALT-PE) as a process measure of achievement. Research Quarterly for Exercise and Sport, 62(3), 319-325.Recuperado de https://shapeamerica.tandfonline.com/doi/abs/10.1080/02701367.1991.10608729\#.W14LINJKjIU

Taylor, S. y Bogdan, H. (1987). Introducción a los métodos cualitativos de investigación. Barcelona, España: Paidós

Thomas, K. \& Thomas, J. (1994).Developing expertise in sport: The relation of knowledge and Performance. International Journal of Sport Psychology, 25, 295-315.Recuperado de https://www.researchgate.net/profile/Jerry_Thomas2/publication/232444616_Developing_expertise_in_ sport_The_relation_of_knowledge_and_performance/links/0c96052ebff462bd9b000000/Developing-exper tise-in-sport-The-relation-of-knowledge-and-performance.pdf

Toro, S., Pazos, J., Vargas, E. y Vega, J. (2016). De la educación global a la biología del conocer: Comprender el sentido de la educación. Estudios pedagógicos (Valdivia), 42(ESPECIAL), 57-73.

Tulviste, P. (1992). Diversidad cultural y heterogeneidad en el pensamiento. Apuntes de psicologia, (35), 5-15. Recuperado de http://europa.sim.ucm.es/compludoc/AA?articuloId $=255215$

Valles, M. (1999). Técnicas cualitativas de investigación social. Reflexión metodológica y práctica profesional. Madrid: Editorial Síntesis, S.A.

Velázquez, R., Hernández, J., Martínez, M. y Martínez de Haro, V. (2011). Educación Física y conocimiento teórico-conceptual: estudio trans-cultural en Argentina, Brasil y España. Revista de Educación, 356. Septiembrediciembre 2011, pp. 653-675. Recuperado de https://repositorio.uam.es/handle/10486/660622

Wertsch, J. (2000). Intersubjectivity and alterity in human communication. Communication: An arena of development, 17-31. Recuperado de https://books.google.cl/books? $\mathrm{hl}=$ es\&lr=\&id=fO9GrixT7XgC\&oi=fnd\&pg=PA17\&dq=Wertsch, +J.+(2000).+Intersubjectivity + and +alterity+in+human+communication.+Communication:+An+arena+of+development, $+17-31 . \&$ ots $=\mathrm{f} 2 \mathrm{l}$ v4cL4J2\&sig=8pqqMWyLBRtfUyWKcNj8BmqwygA\# $\mathrm{v}=$ onepage\&q\& $\mathrm{f}=$ false

Zabala, M., Viciana, J. y Lozano, L. (2002). La planificación de los deportes en la educación física de ESO. Educación Física y deportes. Revista Digital, 8, 48. http://www.efdeportes.com/efd48/eso1.htm

Zabala, V. (2000). "El aprendizaje de los contenidos según su tipología" en La práctica educativa. Cómo enseñar. (7a ed). España: Graó. 